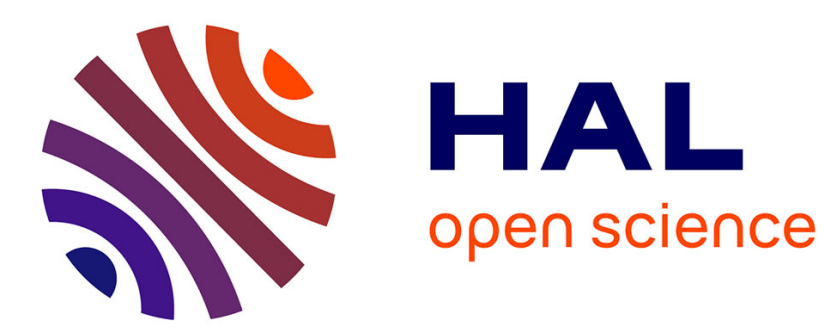

\title{
A Class of Focusing Waves with Controllable Spatial Extension and Directivity
}

Philippe Meton, Andrea Cozza, Florian Monsef

\section{To cite this version:}

Philippe Meton, Andrea Cozza, Florian Monsef. A Class of Focusing Waves with Controllable Spatial Extension and Directivity. IEEE Antennas and Wireless Propagation Letters, 2014, PP (99), 4 p. 10.1109/LAWP.2014.2348935 . hal-01056114

\section{HAL Id: hal-01056114 \\ https://hal-centralesupelec.archives-ouvertes.fr/hal-01056114}

Submitted on 14 Aug 2014

HAL is a multi-disciplinary open access archive for the deposit and dissemination of scientific research documents, whether they are published or not. The documents may come from teaching and research institutions in France or abroad, or from public or private research centers.
L'archive ouverte pluridisciplinaire HAL, est destinée au dépôt et à la diffusion de documents scientifiques de niveau recherche, publiés ou non, émanant des établissements d'enseignement et de recherche français ou étrangers, des laboratoires publics ou privés. 


\title{
A Class of Focusing Waves with Controllable Spatial Extension and Directivity
}

\author{
Philippe Meton and Andrea Cozza, Senior Member, IEEE and Florian Monsef, Member, IEEE
}

\begin{abstract}
Slepian's concentration operator is applied in order to define a set of focusing waves with finite angular spread and the highest spatial focusing possible. These waves can be used as building blocks for the definition of any wave that requires to ensure both angular and spatial resolution, as an optimal trade-off within the bounds imposed by the uncertainty principle. Applications of these results can be expected in the form of focusing waves yielding higher energy efficiency for antenna characterization, EMC radiative-immunity tests, electronic warfare, as well as spatially-resolved beamforming.
\end{abstract}

Index Terms-Focusing waves, plane-wave spectrum, Slepian functions

\section{INTRODUCTION}

Plane waves, or more precisely locally-plane waves, are an essential concept in most test facilities based on the generation of waves impinging on a device under test, as well as in wireless communications. While their appeal is partly motivated by their mathematical and intuitive simplicity, they are not necessarily the best choice. Two drawbacks can be identified; the first one is their lack of spatial resolution, due to their (ideally) infinitely large wavefronts. The second drawback requires recalling that as soon as one realizes that a locally-plane wave is in fact diverging from its source, its intensity decreases while getting away it. In this respect, it cannot be regarded as an energy-efficient choice.

A lack of spatial resolution is also an issue in wireless communications, where radiation over a large region of space can represent a waste of energy, an issue of concern for green communications [1], as well as in electronic warfare, where the need to generate high-intensity fields requires focusing capabilities [2].

The solution to these two drawbacks is the use of focusing waves, which can be designed in such a way as to interfere constructively over a focal plane, where the field intensity will build up to its maximum value, while reducing its transversal extension, thus gaining spatial resolution. The price to pay for focusing waves is a loss of resolution in the direction of arrival. This outcome is inevitable, since angular and spatial behavior are related through a Fourier-transform pair, thus subject to the uncertainty principle [3].

P. Meton is with CEA, DAM, Gramat, F-46500 Gramat, France. A. Cozza and F. Monsef are with the Département de Recherche en Électromagnétisme, Laboratoire des Signaux et Systèmes (L2S), UMR 8506 SUPELEC - Univ Paris-Sud - CNRS, 3 rue Joliot-Curie, 91192 Gif-sur-Yvette, France. Contact e-mail: andrea.cozzalsupelec.fr

Part of the work here reported was funded by the French National Research Agency through the grant ANR-12-ASTR-0005-01, MIMOCHIC project, within the framework of the 2012 ASTRID program.
The question of how to find the optimal trade-off between angular and spatial resolutions is the subject of this paper. A numerical procedure is introduced for the computation of waves featuring optimal angular and spatial resolution. Examples of typical results are presented. For ease of presentation of the concepts involved, our analysis will be restrained to a 2D propagation scenario. Extension to a 3D case is straightforward and does not entail any further complexity.

\section{PWS REPRESENTATION OF A WAVE}

Submitting a device to an impinging wave typically requires ensuring a dominant direction of incidence; this direction is set equal to $\hat{\boldsymbol{y}}$ in the rest of this paper, with no loss of generality. Any time-harmonic electromagnetic wave can be represented as a continuous superposition of plane waves [4], each one characterized by a propagation vector $\boldsymbol{k}=\eta \hat{\boldsymbol{x}}+\xi \hat{\boldsymbol{y}}$, with $\|k\|=k_{o}$, the medium wavenumber; bold symbols stand for vector quantities, hatted ones for unit vectors. The electric field $\boldsymbol{E}(\boldsymbol{r})$ of a wave can thus be expressed as

$$
\boldsymbol{E}(\boldsymbol{r})=\int \mathrm{d} \eta \tilde{\boldsymbol{E}}(\eta) \mathrm{e}^{-\mathrm{j} \eta x} \mathrm{e}^{-\mathrm{j} y \sqrt{k_{o}^{2}-\eta^{2}}}
$$

The function $\tilde{\boldsymbol{E}}(\eta)$, also known as plane-wave spectrum (PWS), is a dual representation of the wave, and corresponds to the complex amplitude of each plane wave contributing to the overall wave $\boldsymbol{E}(\boldsymbol{r})$. The PWS can be related to the electric field over the focal plane $y=0$ as

$$
\tilde{\boldsymbol{E}}(\eta)=\mathcal{F}\{\boldsymbol{E}(x, 0)\},
$$

with $\mathcal{F}\{\cdot\}$ Fourier's transform. The definition (2) of the PWS is fundamental to our analysis, since it allows passing from the spatial distribution of the electric field over the focal plane, to the angular distribution of impinging plane waves, since $\eta=k_{o} \sin \theta$, with $\theta$ defined w.r.t. the $y$ axis. Hereafter, all spatial variables will be regarded as normalized to the working wavelength, so that $k_{o}=2 \pi$.

\section{THE CONCENTRATION OPERATOR}

With the spatial and angular distributions forming a 1D Fourier-transform pair in (2), a solution to the problem of optimal concentration can be found in Slepian and coworkers' work [5], [6]. The problem they faced in the 60's was very similar, as they looked for band-limited signals optimally resolved in time domain. The analogy with the problem of focusing waves implies enforcing band-limitedness in the angular domain, while requiring the highest spatial concentration of energy over the focal plane. A closely related problem 
has been studied more recently in geophysics, especially by Simons [7], in order to generate sets of band-limited spatial distributions confined to arbitrary regions of space.

Slepian's approach to the problem is based on the introduction of the concentration operator $\mathcal{C}$, which applies to a function $g(x)$ as

$$
\mathcal{C} g(x)=\int_{-W}^{W} \mathrm{~d} x^{\prime} D\left(x, x^{\prime}\right) g\left(x^{\prime}\right),
$$

with $[-W, W]$ the portion of the focusing plane over which the wave needs to be concentrated. The kernel

$$
D\left(x, x^{\prime}\right)=\int_{-K}^{K} \mathrm{~d} \eta \mathrm{e}^{-\mathrm{j} \eta\left(x-x^{\prime}\right)}
$$

acts as a sharp band-limiting filter operating over the spectral variable $\eta$. The intuitive idea behind this formal definition is to have this operator letting only spectral components $|\eta| \leq K$ to survive, after having truncated the spatial distribution $g(x)$ over the interval $[-W, W]$. The spectral bound $K$ translates into an angular one as $K=2 \pi \sin \Theta$, with $\Theta$ the maximum angle of incidence allowed. Hence, the reasoning goes, any function $s_{n}(x)$ which is mapped by the operator into itself

$$
\mathcal{C} s_{n}(x)=\lambda_{n} s_{n}(x),
$$

i.e., eigensolutions of (3), must satisfy the two spatial and spectral conditions. Slepian showed [5] that the associated eigenvalues $\lambda_{n}$ correspond to the fraction of energy of $s_{n}(x)$ living over $[-W, W]$, i.e., how concentrated the solution is, with respect to the required truncation interval $[-W, W]$. Ideal solutions are therefore those with $\lambda_{n} \simeq 1$. Here the term energy should be interpreted in the sense of an $L_{2}$ norm applied over a region of space.

The total number $N_{s}$ of perfectly concentrated eigensolutions can be estimated as the sum of all the eigenvalues, hence for our case, according to [7]

$$
N_{s} \simeq \sum_{n=1}^{\infty} \lambda_{n}=\frac{c}{\pi}=2 W \sin (\Theta),
$$

with

$$
c=K W=2 \pi W \sin (\Theta)
$$

for $\Theta \leq \pi / 2$. For $n>N_{s}, \lambda_{n}$ drops very quickly to small values, implying waves that do not focus over the interval $x \in[-W, W]$, but rather outside it.

In order to directly apply these ideas to our problem, we need to look for scalar solutions. One way of doing so is to consider TM impinging waves and look for electric field distributions $\boldsymbol{E}_{n}(x, 0)=E_{n}(x, 0) \hat{z}$ such that $\mathcal{C} E_{n}(x, 0)=$ $\lambda_{n} E_{n}(x, 0)$. The TE case can be solved in a similar way, by looking for a scalar solution for the magnetic field, which would then take the shape $\boldsymbol{H}_{n}(x, 0)=H_{n}(x, 0) \hat{\boldsymbol{z}}$; the PWS allows to pass from electric to magnetic field by simply recalling that $\tilde{\boldsymbol{H}}(\eta)=\zeta^{-1} \hat{\boldsymbol{k}} \times \tilde{\boldsymbol{E}}(\eta)$, with $\zeta$ the wave impedance of the propagation medium. In the following, Slepian solutions will be referred to as $s_{n}(x)$.

While spatial and angular bounds can be set independently, the existence of solutions to (3) entirely depends on the value c. Indeed, it was proven in [5] that it also controls the degree of concentration

$$
\lambda_{n}(c)=\frac{2 c}{\pi}\left[R_{0 n}^{(1)}(c, 1)\right]^{2},
$$

where $R_{m n}^{(i)}(c, t)$ are radial prolate spheroidal wave functions (PSF), as defined according to Flammer's notations [8]. To a required minimum $\lambda_{m}$, corresponds a value $c_{m}^{p}$, obtained by inverting (8) for a given order $p$, such that for any $c \geq c_{m}^{p}$ Slepian solutions will have $\lambda_{n} \geq \lambda_{m}, \forall n \leq p$. Eq. (7) can then be applied in order to know how spatial and angular resolutions should relate in order to ensure that $c \geq c_{m}$; it also shows that increasing the maximum angular spread does not always lead to a stronger focusing, because of the nonlinear behavior of the sine function.

Unfortunately, the PSFs in (8) cannot be evaluated easily. Their numerical computation are still a matter of ongoing research, as discussed in [9], [10]. In practice, evaluation of (8) are based on tables published in Slepian's work and cannot be simply approximated. The same problem exists when looking for eigensolutions of (3), since they are again given by PSFs.

In this respect, we prefer adopting a simpler and more pragmatic approach, approximating (3) by a matrix operator; in a similar way Simons also looked for truncated modal expansions of the concentration operator [7]. We apply Galerkin's method [11] in order to solve the integral equation (3), i.e., by expressing unknown solutions into a linear combination of orthogonal basis functions. A suitable choice is to use plane waves as basis functions, which allow enforcing straightforwardly that solutions be band-limited by selecting only those propagating along directions $|\theta| \leq \Theta$.

Since it is often preferable to generate waves that are evensymmetric with respect to the main direction of arrival, it is necessary to enforce the same amplitude for all pairs of plane waves propagating along specular directions. As a result, generic solutions $s_{n}(x)$ will be sought with the following expansion

$$
s_{n}(x)=\sum_{p=0}^{N_{b}} \tilde{s}_{n}\left(\eta_{p}\right) \cos \left(\eta_{p} x\right) \Delta \eta,
$$

where $N_{b}$ is the number of pairs of plane waves by which the solutions are approximated. In (9), the spectral variable $\eta$ is discretized into $2 N_{b}+1$ samples $\left\{\eta_{p}\right\}$, uniformly distributed over the interval $[-K, K]$.

Even-symmetric pairs of plane waves are also used as test functions in Galerkin's method, corresponding to a collocation scheme in the spectral domain. With this choice in mind, the approximate discrete version of (5) is

$$
\sum_{p=0}^{N_{b}} \beta_{p q} \tilde{s}_{n}\left(\eta_{p}\right)=\lambda_{n} \sum_{q=0}^{N_{b}} \alpha_{p q} \tilde{s}_{n}\left(\eta_{q}\right)
$$

with

$$
\beta_{p q}=2 W\left\{\operatorname{sinc}\left[W\left(\eta_{p}-\eta_{q}\right)\right]+\operatorname{sinc}\left[W\left(\eta_{p}+\eta_{q}\right)\right]\right\}
$$

the discretized version of the concentration operator and

$$
\alpha_{p q}=\pi / \Delta \eta \begin{cases}\delta_{p q} & p+q>0 \\ 2 & p=q=0\end{cases}
$$




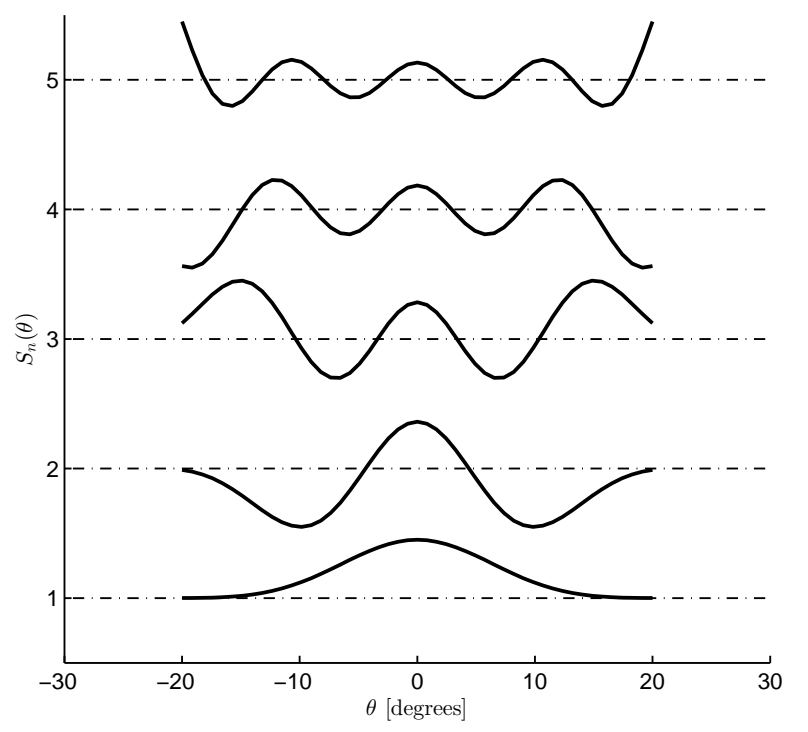

Fig. 1: PWS of the first 5 eigensolutions of (5), for the case $W=5, \Theta=20$ degrees.

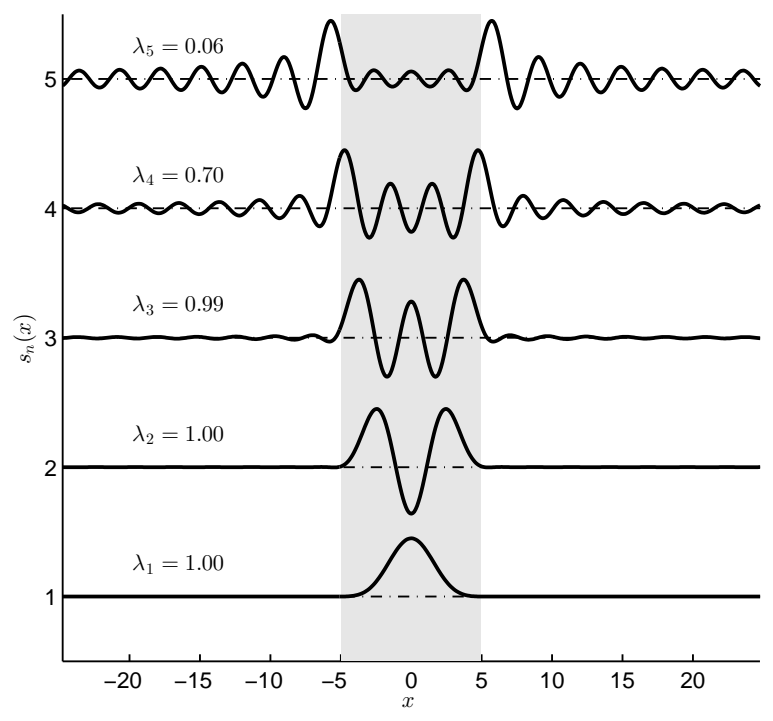

Fig. 2: Spatial distributions associated to the spectra shown in Fig. 1. The spatial energy concentrations, corresponding to the eigenvalues of (5) are shown for each solution. The shaded region stands for the interval over which the solutions should be bound.

the matrix of mutual energies of the cosine basis functions, obtained by projecting the $p$ th function over the $q$ th. Assembling the $N_{b}$ equations in (10) into a matrix form yields a generalized eigenproblem

$$
\boldsymbol{\beta} \tilde{\boldsymbol{s}}_{n}=\lambda_{n} \boldsymbol{\alpha} \tilde{\boldsymbol{s}}_{n},
$$

whose eigenvectors are a set of discrete PWS $\tilde{s}_{n}\left(\eta_{p}\right)$ of focusing waves.

\section{NUMERICAL RESULtS}

In order to illustrate the ideas so far discussed, we consider the case of a focal region bound by $W=5$ and $\Theta=20$ degrees. Since the solutions obtained from (13) are based on discrete PWSs, the spatial distribution will be characterized by a period $P=2 \pi / \Delta \eta$. Therefore, the number of basis functions in (9) can be chosen by enforcing $P \gg W$, hence $N_{b} \gg$ $2 W \sin \Theta$. This constraint actually coincides with the need for a number of basis functions much larger than the number of most concentrated solutions expected for (13).

The results in Fig. 1 are the PWS of the first 5 eigensolutions of (5), plot from the most concentrated (no. 1) to the least one (no. 5). The associated energy concentrations were found to be $1.00,1.00,0.99,0.70$ and 0.06 . The expected number of solutions with optimal concentration was estimated to be 3 , as given by (6), which appears to be quite accurate. Identical results would be obtained for any combination of $W$ and $\Theta$ s.t. (7) is unmodified. As expected, the concentration of higherorder solutions falls quickly to very weak values; the existence of a finite number of confined solutions shows the importance of Slepian's approach: by computing the very limited set of possible solutions, it is possible to know beforehand what configuration will make it possible to satisfy stringent requirements on directivity and spatial focusing.

These PWSs produce spatial distributions over the focal plane that are shown in Fig. 2, while their field distributions over the rest of the space can be computed by means of (1) and are shown in Fig. 3. These results highlight that solutions to the concentration operator (3) are not required to fill in the allowed region $[-W, W]$, but are merely required not to trespass it. Functions of increasing order cover an incrementally larger portion of $[-W, W]$.

These waves, which could be referred to as Slepian waves, constitute building blocks that can be used for defining any focusing wavefront with angular and spatial resolution. In case one were interested in producing, e.g., a flat focalplane distribution limited to the region $[-W, W]$, its PWS could not possibly be angularly-limited, and would further require reactive components to produce the sharp transitions at $x= \pm W$. Slepian solutions can be used in order to enforce the double constraint of spatial and angular resolution, e.g., for a TM wave, approximating the desired flat focal-region profile $E_{t}(x, 0)$ by

$$
E(x, 0)=\sum_{n=1}^{N} d_{n} E_{n}(x, 0),
$$

where only the first $N \leq N_{s}$ solutions estimated from (6) are considered, among the most concentrated ones.

The coefficients $d_{n}$ can be computed by projecting $E_{t}(x, 0)$ over this set of Slepian solutions, as they form an orthogonal set. Some of the resulting waves are shown in Fig. 4. The number of Slepian waves from the previous example was limited to the first three, characterized by $\lambda_{i} \simeq 1$; first four weights $d_{n}=\{2.29,1.55,1.25,0.71\}$. As expected, smooth transitions to zero appear instead of the sharp transitions of the target distribution $E_{t}(x, 0)$. Moreover, Paley-Wiener theorem requires that, in order to have a compact spatial distribution, 

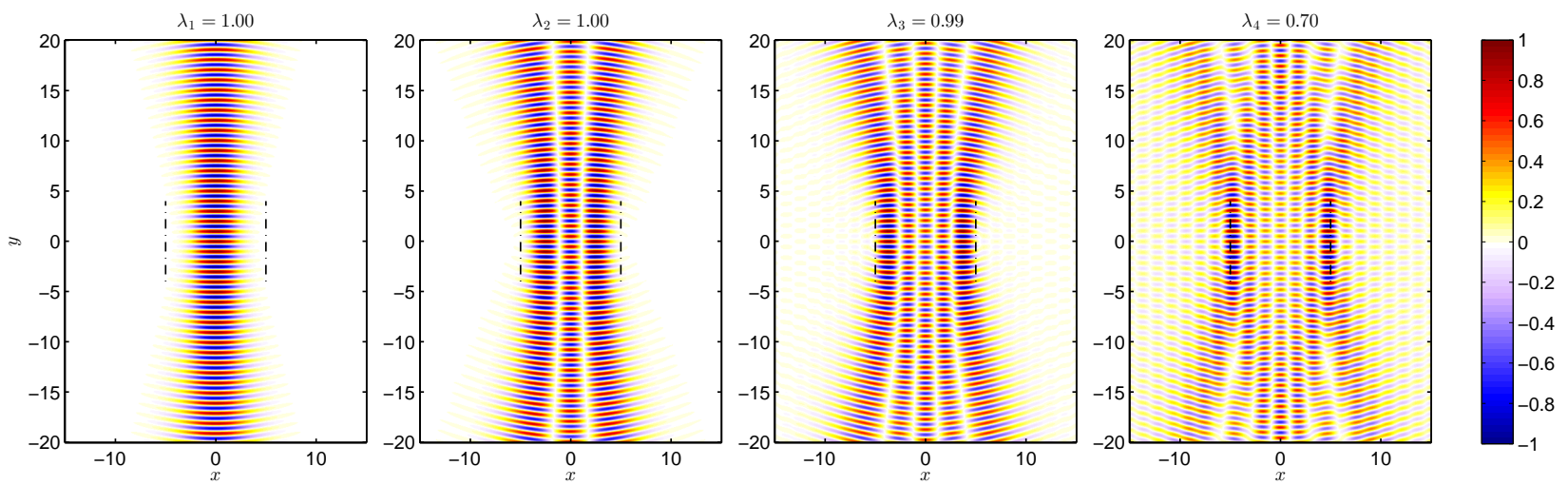

Fig. 3: The first four waves obtained with the proposed procedure, for the case of $W=5$, for $\Theta=20$ degrees. The two vertical dashed lines mark the bounds imposed at the focal plane. Distances are expressed in wavelengths, amplitudes are peak normalized.
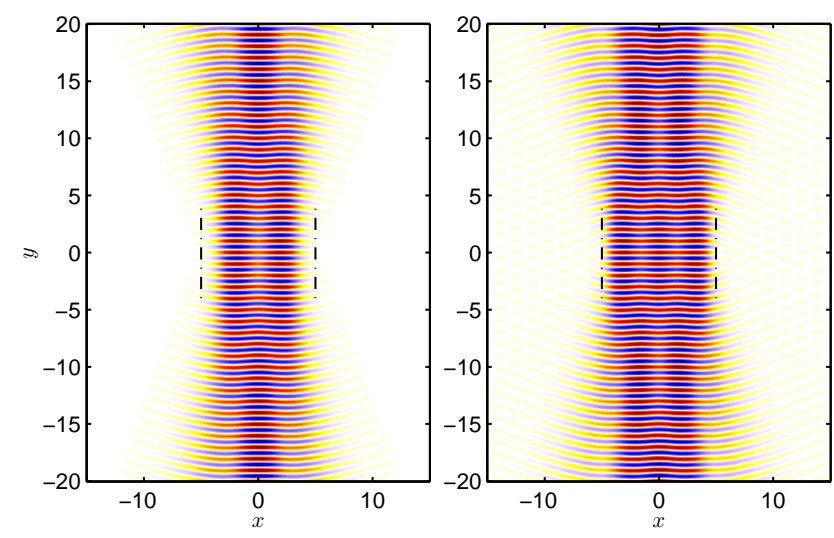

Fig. 4: Results obtained by approximating a flat-amplitude focal region, with the first two (left) and three (right) Slepian waves shown in Fig. 3. The color scales are identical.

its Fourier transform must decay exponentially. Fig. 5 allows appreciating this property: as the number of Slepian waves involved increases, the resulting angular distributions decay ever less smoothly; for $N=3$, the PWS starts being truncated. Smoother PWSs inevitably differ from the target PWS, by a degree that depends on the final concentration required.

\section{Conclusions}

Slepian's work provides a simple and elegant solution to the problem of defining focusing waves with both angular and spatial resolution. A method-of-moment approximation, based on a Fourier series representation of the focal-plane field distribution was applied to find approximate solutions to the concentration operator. The set of solutions thus defined can be used as building blocks in the definition of waves of potential interest in applications such as EMC testing and electronic warfare.

\section{REFERENCES}

[1] B. Wang, Y. Wu, F. Han, Y.-H. Yang, and K. Liu, "Green wireless communications: A time-reversal paradigm," Selected Areas in Coтmu-

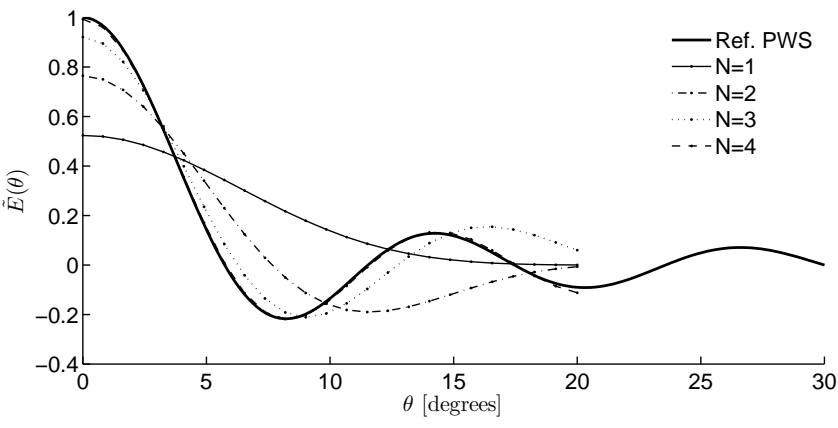

Fig. 5: Angular distributions of the PWSs involved in the generation of a compact constant-amplitude focal region with $W=5$; four Slepian approximations are shown enforcing $\Theta=20$ degrees, together with the PWS corresponding to a flat focal distribution with sharp transitions.

nications, IEEE Journal on, vol. 29, no. 8, pp. 1698-1710, September 2011.

[2] R. Poisel, Antenna Systems and Electronic Warfare Applications. Artech House, 2012.

[3] L. Cohen, Time-frequency analysis: theory and applications. PrenticeHall, Inc., 1995.

[4] P. Clemmow, The plane wave spectrum representation of electromagnetic fields. Pergamon Press (Oxford and New York), 1966.

[5] D. Slepian and H. O. Pollak, "Prolate spheroidal wave functions, Fourier analysis and uncertainty -I," Bell System Technical Journal, vol. 40, no. 1 , pp. 43-63, 1961.

[6] D. Slepian, "On bandwidth," Proceedings of the IEEE, vol. 64, no. 3, pp. 292-300, 1976.

[7] F. J. Simons, "Slepian functions and their use in signal estimation and spectral analysis," in Handbook of Geomathematics. Springer, 2010, pp. 891-923.

[8] C. Flammer, Spheroidal Wave Functions. Stanford University Press, 1957.

[9] G. Walter and T. Soleski, "A new friendly method of computing prolate spheroidal wave functions and wavelets," Applied and Computational Harmonic Analysis, vol. 19, no. 3, pp. 432-443, 2005.

[10] A. Karoui and T. Moumni, "New efficient methods of computing the prolate spheroidal wave functions and their corresponding eigenvalues," Applied and Computational Harmonic Analysis, vol. 24, no. 3, pp. 269289, 2008.

[11] L. V. Kantorovich and V. I. Krylov, "Approximate methods of higher analysis," New York, 1958. 